\title{
Manejo de las lesiones anexiales en el embarazo y actualización del abordlaje laparoscópico
}

\section{Management of adnexal masses in pregnancy and an update of the laparoscopic approach}

\section{Raimundo Avilés ${ }^{1,2,3 *}$, María C. Cornejo 1,2, Constanza Norambuena ${ }^{1,2}$, Demetrio Larrain 4 , Dusan Bader², Francisca Álvarez ${ }^{2}$ y Gabriela Araya²}

${ }^{1}$ Departamento de Obstetricia y Ginecología, Hospital Luis Valentín Ferrada El Carmen, Maipú; ${ }^{2}$ Departamento de Obstetricia y Ginecología, Universidad Finis Terrae; ${ }^{3}$ Departamento de Obstetricia y Ginecología, Clínica Las Condes; ${ }^{4}$ Departamento de Obstetricia y Ginecología, Clínica Santa María, Santiago, Chile

\section{Resumen}

El manejo del tumor anexial en embarazadas sigue siendo un desafío. El objetivo del siguiente trabajo es realizar una revisión bibliográfica, en la que se abordarán las diferentes alternativas en cuanto a diagnóstico y manejo del tumor anexial en embarazadas, así como también indicaciones de abordaje quirúrgico y recomendaciones para una cirugía segura. Los tumores anexiales en embarazadas son poco frecuentes, y la mayoría son benignos y tipo funcionales. La ecografía ha sido fundamental para lograr diferenciar su carácter benigno o maligno. El manejo puede ser expectante o quirúrgico. El manejo quirúrgico se reserva para ciertas características de las lesiones anexiales. En cuanto a la vía operatoria, la literatura apoya la laparoscopia mostrando que existen múltiples beneficios al compararla con la laparotomía. Para el abordaje quirúrgico existen opciones en cuanto a la técnica, siendo estas anexectomía o quistectomía. Resulta fundamental tener consideraciones especiales en la técnica debido a los cambios fisiológicos de las embarazadas, como por ejemplo la altura uterina y el tamaño del tumor anexial. A modo de conclusión, el manejo de los tumores anexiales en el embarazo sigue siendo controversial y se extrapola principalmente basándose en la literatura de pacientes no gestantes u otros procedimientos quirúrgicos en embarazadas.

Palabras clave: Lesión anexial. Tumor anexial. Laparoscopia. Embarazada. Ecografía ginecológica.

\section{Abstract}

Nowadays the management of the adnexal tumors in pregnant women is still a challenge. The purpose of this article is to perform a bibliographic review and present the differential diagnosis, management, and surgical approaches for the women in this condition. Adnexal tumors in pregnant women are rare, most of them are benign corresponding to functional cysts. In order to differentiate benign from malignant tumors, Ultrasonography has been one of the most important imaging advances. The management can be either expectant or surgical. Surgical management is referred for tumors with certain specific characteristics. In relation to surgical management, the literature supports laparoscopy, showing greater benefits in comparison to laparotomy. There are different options for this kind of approach. It's mandatory to have special considerations in the technique due to the physiological changes in pregnant women, some examples are the uterine and the tumor size. The management of the adnexal tumors in pregnancy is still controversial, it's based on studies of non-pregnant patients or other kinds of surgeries in pregnant women.

Keywords: Adnexal mass. Adnexal tumor. Laparoscopy. Pregnancy. Gynecologic ultrasonography.

Correspondencia:

${ }^{*}$ Raimundo Avilés

E-mail: raviles@ uft.cl
Fecha de recepción: 08-03-2021

Fecha de aceptación: 09-12-2021

DOI: 10.24875/RECHOG.M21000036
Disponible en internet: 07-02-2022 Rev Chil Obstet Ginecol. 2021;86(6):573-582 www. rechog.com 0048-766X / ๑ 2021 Sociedad Chilena de Obstetricia y Ginecología. Publicado por Permanyer. Éste es un artículo open access bajo la licencia CC BY-NC-ND (https://creativecommons.org/licenses/by-nc-nd/4.0/). 


\section{Introducción}

Actualmente es cada vez más frecuente la presencia de tumores anexiales en el embarazo, la mayoría diagnosticados de forma incidental al realizar el cribado ecográfico durante el primer trimestre o relacionado con el estudio de síntomas ${ }^{1,2}$. El manejo se ha convertido en un desafío, siendo necesario tener conocimientos tanto de las herramientas diagnósticas y terapéuticas como de sus riesgos.

Es fundamental definir aquellas situaciones en las cuales se puede realizar un manejo expectante frente a uno quirúrgico, con el fin de obtener los mejores resultados tanto para la madre como para el feto. En este sentido, la laparoscopia se posiciona como una buena alternativa de vía de abordaje en aquellas que tienen indicación de manejo quirúrgico, al presentar beneficios como menos días de hospitalización, menor sangrado intraoperatorio y menor tasa de trabajo de parto prematuro, entre otros ${ }^{3-5}$.

El objetivo del siguiente trabajo es realizar una revisión bibliografía acerca de los tumores anexiales en pacientes embarazadas. Se abordarán las diferentes alternativas en cuanto a diagnóstico, manejo, indicaciones de abordaje quirúrgico y recomendaciones para una cirugía segura.

\section{Definición y epidemiología}

Una lesión anexial es un aumento de volumen abdomino-pélvico, de origen ginecológico o extraginecológico $^{6}$. En esta revisión nos enfocaremos en las ginecológicas, que comprenden las trompas uterinas, los ovarios y los parametrios. Estas pueden crecer, comprometer y afectar cualquiera de dichas estructuras, siendo la gran mayoría tumores benignos ${ }^{3,7}$.

La prevalencia de tumores anexiales en embarazadas se describe entre un $0,05 \%$ y un $3,2 \% 8,9$. Este porcentaje ha ido en aumento en el último tiempo, debido al incremento de ecografías sistemáticas en el embarazo ${ }^{7,8,10}$, siendo más frecuente el diagnóstico en los primeros dos trimestres de gestación y que el carácter de la lesión sea benigno, principalmente de origen funcional ${ }^{11}$.

En relación con los tumores ováricos orgánicos se encuentran los teratomas y los tumores epiteliales; sin embargo, no está claramente definido en la literatura cuál es el más frecuente ${ }^{3,12,13}$.

En cuanto a las lesiones malignas, estas corresponden a un $3.6 \%$ a $6.8 \%$ de las lesiones ováricas persistentes en las embarazadas ${ }^{8}$, misma proporción que se presenta en las pacientes no gestantes. En las embarazadas, el cáncer epitelial de ovario es el tipo más común de las neoplasias malignas, describiéndose en aproximadamente el $50 \%$ de los casos; el $30 \%$ son de células germinales y tumores del estroma, y el $20 \%$ otras afecciones tumorales como sarcomas y metástasis, entre otras ${ }^{14}$.

\section{Diagnóstico}

Con el desarrollo de la ecografía obstétrica ha aumentado el diagnóstico incidental de lesiones anexiales en embarazadas ${ }^{15}$. La mayoría de estas lesiones son asintomáticas y presentan resolución espontánea en el $51-70 \%$ de los casos, mientras que sufren complicaciones menores el $2 \%{ }^{16}$. Lograr distinguir entre lesiones funcionales, benignas y malignas ha sido un constante desafío.

Los tipos de lesiones se detallan en la tabla 1. Los hallazgos que orientan con mayor probabilidad a una lesión maligna se indican en la tabla 212,17.

Es fundamental la evaluación por ecografistas expertos, quienes ayudan a discernir sobre el origen de las lesiones. En caso de no contar con experiencia, actualmente se recomienda aplicar la terminología IOTA para describir y usar algoritmos para evaluar la probabilidad de malignidad de una lesión, o referir a un ecografista experto. Los algoritmos del grupo IOTA se encuentran ampliamente validados en premenopáusicas y posmenopáusicas, pero no en embarazadas. Se aconseja iniciar con las simple rules que permiten caracterizar un $76-89 \%$ de las lesiones anexiales y son de fácil aplicación, por lo que pueden ser utilizadas por ecografistas menos entrenados, considerando las características expuestas en la tabla 3. Estas presentan una sensibilidad del $93 \%$ y una especificidad del $95 \%{ }^{18}$. Sin embargo, un $25 \%$ de las lesiones no pueden caracterizarse y deben ser evaluadas por un ecografista experto 0 aplicar otros modelos predictivos, como LR1, LR2 o ADNEX ${ }^{19}$.

Al evaluar una lesión anexial, la presencia de una o más características benignas aumenta la probabilidad de que lo sea, sucediendo lo mismo con las características malignas. Sin embargo, las lesiones que presenten ambas características no pueden clasificarse y deben ser evaluadas con otro algoritmo 0 ser derivadas a un ecografista con experiencia, cuya evaluación tiene una sensibilidad del $95 \%$ y una especificidad del $94 \%{ }^{20}$. 
Tabla 1. Tipos de lesión anexial sospechosa según hallazgos ecográficos

\begin{tabular}{|c|c|c|c|c|c|}
\hline Lesiones & Funcionalidad & Loculado & Ecogenicidad & Otras & Manejo \\
\hline Cuerpo lúteo quístico & Funcional & $\begin{array}{l}\text { Unilocular, } \\
\text { quística }\end{array}$ & Hiperecogénica & $\begin{array}{l}\text { Vascularización periférica al } \\
\text { doppler }\end{array}$ & $\begin{array}{l}\text { Probable resolución } \\
\text { espontánea }\end{array}$ \\
\hline Quiste folicular & Funcional & $\begin{array}{l}\text { Unilocular, } \\
\text { quística }\end{array}$ & Anecogénica & Sin vascularización al doppler & $\begin{array}{l}\text { Probable resolución } \\
\text { espontánea }\end{array}$ \\
\hline Quiste hemorrágico & Funcional & $\begin{array}{l}\text { Unilocular, } \\
\text { quística }\end{array}$ & Hiperecogénica & $\begin{array}{l}\text { Trabecular, vascularización } \\
\text { periférica al doppler color. }\end{array}$ & $\begin{array}{l}\text { Probable resolución } \\
\text { espontánea }\end{array}$ \\
\hline Endometrioma & No Funcional & Unilocular & Hiperecogénica & $\begin{array}{l}\text { Homogénea, bajo nivel, vidrio } \\
\text { esmerilado }\end{array}$ & $\begin{array}{l}\text { Benigna, seguimiento } \\
\text { ecográfico }\end{array}$ \\
\hline Teratoma & No Funcional & Unilocular & Ecomixta & Sombra acústica & $\begin{array}{l}\text { Benigna, seguimiento } \\
\text { ecográfico }\end{array}$ \\
\hline Quiste paraoóforo & No Funcional & Unilocular & Anecogénica & Adyacente a ovario & $\begin{array}{l}\text { Benigna, seguimiento } \\
\text { ecográfico }\end{array}$ \\
\hline Hidrosalpinx & No Funcional & Unilocular & Anecogénica & $\begin{array}{l}\text { Paraovárico, elongado, } \\
\text { tabiques incompletos }\end{array}$ & $\begin{array}{l}\text { Benigna, seguimiento } \\
\text { ecográfico }\end{array}$ \\
\hline
\end{tabular}

Tabla 2. Hallazgos ecográficos que orientan a malignidad

Hallazgos ecográficos que orientan a malignidad
Lesiones no uniloculares
Presencia de áreas sólidas
Lesiones vascularizadas
Bordes irregulares
Aumento de tamaño al comparar dos imágenes
Tamaño mayor a $10 \mathrm{~cm}$
Bilateralidad
Ascitis

Tabla 3. Características benignas y malignas en base a las Simple rules

\begin{tabular}{|l|l|}
\hline $\begin{array}{l}\text { Características benignas de } \\
\text { las Simple Rules }\end{array}$ & $\begin{array}{l}\text { Características malignas de } \\
\text { las Simple Rules }\end{array}$ \\
\hline $\begin{array}{l}\text { B1 Lesión unilocular } \\
\text { B2 Componente sólido < de } 7 \\
\text { mm }\end{array}$ & M1 Tumor sólido irregular \\
\hline $\begin{array}{l}\text { B3 Sombra acústica } \\
\text { B4 Lesión multilocular<de } 10\end{array}$ & $\begin{array}{l}\text { M3 Al menos 4 estructuras } \\
\text { cm }\end{array}$ \\
\hline $\begin{array}{l}\text { B5 Ausencilares multilocular } \\
\text { vascularización al Doppler } \\
\text { color }\end{array}$ & $\begin{array}{l}\text { M5 Vascularización abundante, } \\
\text { Score 4 al Doppler color }\end{array}$ \\
\hline
\end{tabular}

En caso de presentar dudas se puede realizar una resonancia magnética que determina malignidad y caracteriza lesiones anexiales con alta precisión ${ }^{12}$. Sus ventajas son el amplio campo de visión, la evaluación de ascitis y linfadenopatías, el alto contraste de los tejidos blandos y la ausencia de radiación ionizante con posibles efectos nocivos en el feto ${ }^{21}$. El uso de medios de contraste solo debe indicarse si el diagnóstico implica mayores beneficios que riesgos ${ }^{22}$. La tomografía computarizada de abdomen tiene mayor accesibilidad, evitando retrasar el diagnóstico en caso de patologías agudas. Si bien produce radiación, con posibles complicaciones en el feto, presenta bajo riesgo para este ${ }^{22}$.

Sobre los marcadores tumorales, el CA-125 ha demostrado que fluctúa ampliamente en el embarazo y se duda de que sea un marcador significativo de malignidad ovárica en estos casos $^{23}$, pudiendo encontrarse aumentado hasta en un $16 \%$ de las pacientes gestante ${ }^{24}$. Por tanto, no es apropiado para distinguir lesiones anexiales benignas o malignas.

\section{Complicaciones}

Se describen complicaciones como la torsión anexial, la ruptura del quiste y la impactación pélvica con obstrucción del parto ${ }^{11,13}$, con una tasa de ocurrencia del $2 \%$ al $5.7 \% \%^{8,14}$.

Ciertas características de las lesiones, como el tamaño y su tasa de crecimiento, sumado al tamaño del útero, son factores que aumentan la probabilidad de torsión anexial durante el embarazo ${ }^{23}$. En general, cuanto mayor es el tamaño de la lesión más aumenta el riesgo de complicaciones, independientemente de su carácter ${ }^{11}$. 
La torsión ovárica es una de las complicaciones más comunes en el embarazo, siendo más frecuente en caso de tumores entre los 6 y $10 \mathrm{~cm}^{13,25}$. Al comparar población embarazada y no gestante, la literatura describe un $1 \%$ más de riesgo de torsión en el primer grupo ${ }^{11,23}$. Principalmente, entre las 10 y las 17 semanas de gestación el riesgo disminuye a medida que avanzan las semanas, siendo menos del $6 \%$ después de las 20 semanas ${ }^{25}$. Esto se debe al aumento del tamaño del útero, que limita la movilización de otros órganos ${ }^{11}$.

Se ha descrito también que los embarazos concebidos por técnicas de reproducción asistida tienen más riesgo de torsión, dada la asociación con hiperestimulación ovárica ${ }^{13}$.

\section{Manejo \\ Generalidades}

Posterior al diagnóstico hay que determinar qué manejo se realizará: expectante o quirúrgico ${ }^{11}$.

El manejo expectante tiene como beneficios evitar el procedimiento quirúrgico y sus complicaciones ${ }^{13}$, pero presenta riesgos, como la ocurrencia de complicaciones propias de los tumores, como lo son la torsión o la ruptura ${ }^{11,13}$.

Si la mujer no tiene síntomas y en la ecografía hay signos de benignidad, se podría mantener una conducta expectante sin que esto implique mayores riesgos para la madre y el feto ${ }^{11,26-28}$. La mayoría de las lesiones funcionales se resolverán de manera espontánea antes de las 16 semanas, por lo que se plantea seguimiento cada 2-4 semanas. Ante lesiones probablemente malignas o sintomáticas, se sugiere manejo quirúrgico inmediato.

\section{Manejo quirúrgico}

En caso de realizar un manejo quirúrgico, la edad gestacional óptima es a principios del segundo trimestre, entre las semanas 16 y 18 , cuando ya las lesiones anexiales funcionales hayan desaparecido, la organogénesis se haya completado y disminuya el riesgo de aborto. Si el procedimiento se realiza en estas semanas, el riesgo de aborto es menor comparado con el primer trimestre, con un límite superior para realizar el procedimiento de 28 semanas $^{28}$.

En caso de presentarse torsión o ruptura de una lesión anexial, la intervención debe realizarse independientemente del momento del embarazo ${ }^{26}$.

En cuanto a la técnica quirúrgica, se puede realizar por laparotomía o por laparoscopia. Tanto en mujeres gestantes como en no embarazadas, la técnica preferida es la laparoscópica. En esta es fundamental tener algunas consideraciones especiales, las cuales detallaremos más adelante en la descripción de la técnica laparoscópica8.

Al comparar ambas técnicas, la literatura favorece a la laparoscopia por un menor riesgo de parto prematuro, menor pérdida sanguínea y menor estadía hospitalaria, sin diferencias significativas en cuanto al riesgo de aborto y la duración de la cirugía ${ }^{2,3}$. La cirugía laparoscópica se asocia a menor dolor posoperatorio y menos tiempo hasta la deambulación, disminuyendo el riesgo tromboembólico ${ }^{11}$. En las embarazadas genera beneficios similares en otros procedimientos, como apendicectomía o colecistectomía ${ }^{3}$.

El abordaje laparoscópico privilegia realizar la menor alteración posible de la anatomía y la fertilidad de la mujer, como en los casos de quistectomía ${ }^{29}$. En general, la quistectomía se recomienda frente a la presencia de quistes simples, presunción benigna y vitalidad del tejido ovárico ${ }^{29}$.

Si se realiza una quistectomía, se recomienda extirpar el quiste intacto, lo que facilita su disección y previene efectos adversos secundarios a su rotura, como la peritonitis química. Recomendamos ubicar debajo de la lesión una bolsa de extracción endoscópica para evitar el contacto del contenido del quiste con la cavidad abdominal, en caso de romper accidentalmente la pared de este, lo que ocurre con frecuencia en los teratomas, los endometriomas y los quistes de gran tamaño. Es fundamental lograr la extracción completa de la cápsula y realizar una coagulación selectiva del tejido ovárico ${ }^{30}$.

Se recomienda la anexectomía para el manejo de lesiones de aspecto potencialmente maligno, con necesidad de posterior estudio anatomopatológico ${ }^{30,31}$.

En caso de torsión anexial no es inminente realizar una anexectomía, independientemente de cómo se observe el tejido ovárico (necrosis, edema y hemorragia) en la cirugía. Desde el punto de vista histopatológico se ha evidenciado posible parénquima susceptible de recuperación una vez destorcido el ovario, con una tasa del $88-100 \%^{32}$.

Los factores que hay que tener en cuenta al decidir la técnica son la edad de la paciente, el tamaño del tumor y las posibles dificultades técnicas ${ }^{30}$.

\section{Actualización de la técnica laparoscópica}

\section{Fisiología del embarazo y cirugía laparoscópica}

El embarazo no se considera una contraindicación para la cirugía laparoscópica ${ }^{33}$, y en comparación con 


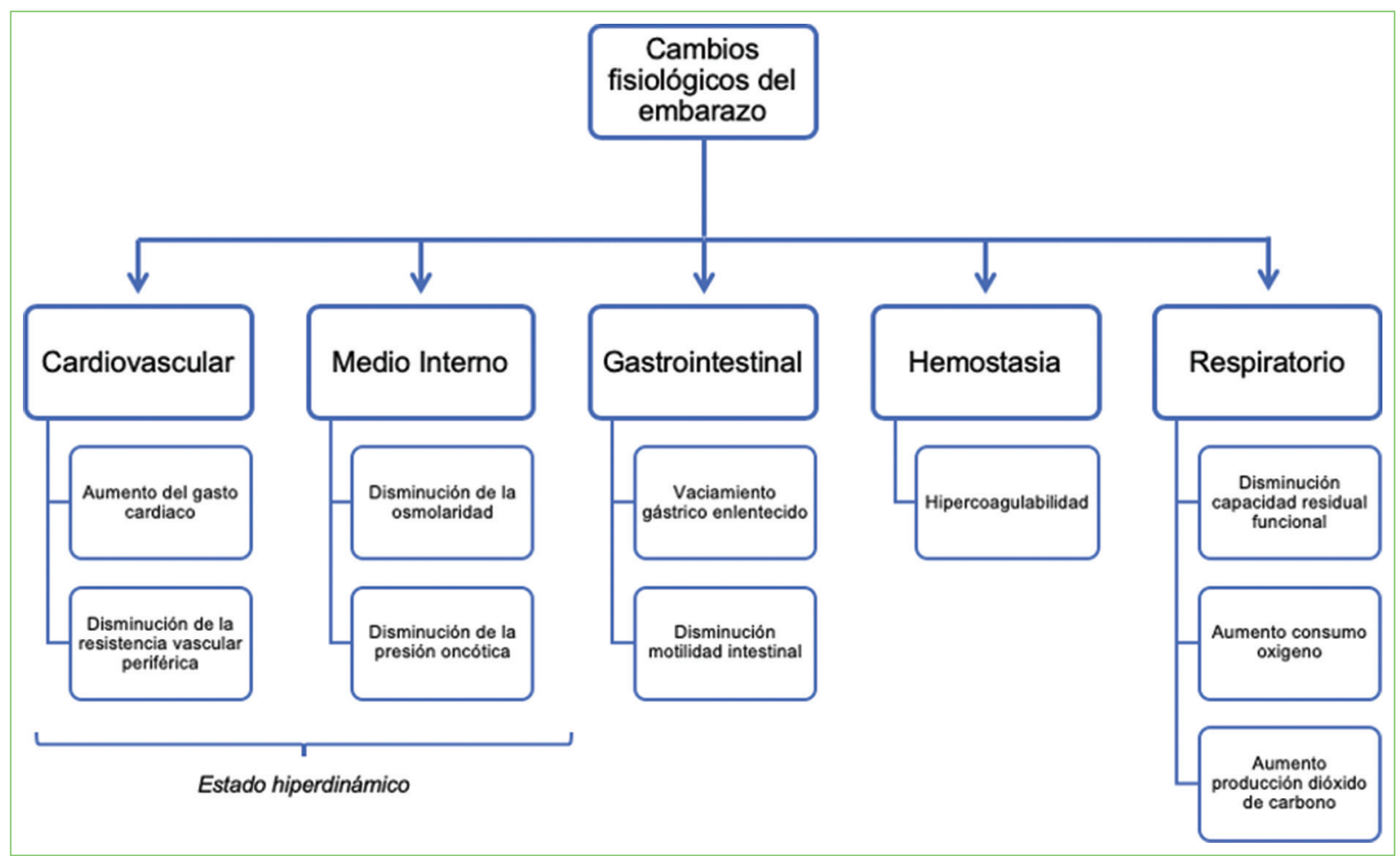

Figura 1. Cambios fisiológicos que ocurren durante el embarazo y qué se podría ver afectado por la cirugía laparoscópica ${ }^{26}$.

la cirugía abierta no hay mayor riesgo para la madre ni para el feto, siendo considerado un abordaje apropiado si se dispone del equipamiento adecuado y de experiencia ${ }^{2}$.

Es fundamental conocer los cambios fisiológicos maternos y cómo interactúan con la cirugía laparoscópica. Nos centraremos en los sistemas que se ven afectados por la técnica laparoscópica. En la figura 1 se muestran los cambios fisiológicos según el sistema afectado ${ }^{26}$.

Ya teniendo claros los cambios que ocurren en la embarazada, analizaremos los efectos de la cirugía sobre estos. Hay tres grandes ejes que son parte de la cirugía laparoscópica y que influyen en los distintos sistemas: el neumoperitoneo, la posición supina y el manejo de la vía aérea (Figs. 2 a 4) ) $^{26}$.

\section{Técnica laparoscópica}

Hay que considerar y definir la edad gestacional para la intervención en las cirugías electivas y evaluar la experiencia del equipo médico para una planificación adecuada, logrando así minimizar al máximo los tiempos operatorios y los riesgos ${ }^{26,34}$.

Consideraciones intraoperatorias:
- Posicionar a la paciente siempre horizontal (no Trendelenburg) al momento de ingresar a la cavidad peritoneal.

- Utilizar medias antiembólicas para disminuir el riesgo tromboembólico materno ${ }^{34,35}$.

- Uso de sonda de Foley y de sonda nasogástricauorogástrica para vaciar la vejiga y el estómago ${ }^{34}$.

- Está contraindicado el uso de un manipulador uterino $y$ de instrumentos intravaginales ${ }^{36}$.

- Al momento de la instalación laparoscópica no hay una técnica de preferencia, utilizándose la insuflación con aguja de Veress, la entrada directa o la técnica abierta, como la descrita por Hasson ${ }^{26,36}$. La técnica quirúrgica se explicará de forma más detallada más adelante, en el posicionamiento de trocares.

- Para el posicionamiento durante el procedimiento mismo existen variaciones según el trimestre. En el primer trimestre es similar al de la mujer no gestante, y en el segundo y tercer trimestres en decúbito lateral izquierdo, o bien se recomienda lateralizar la mesa $^{34}$. 


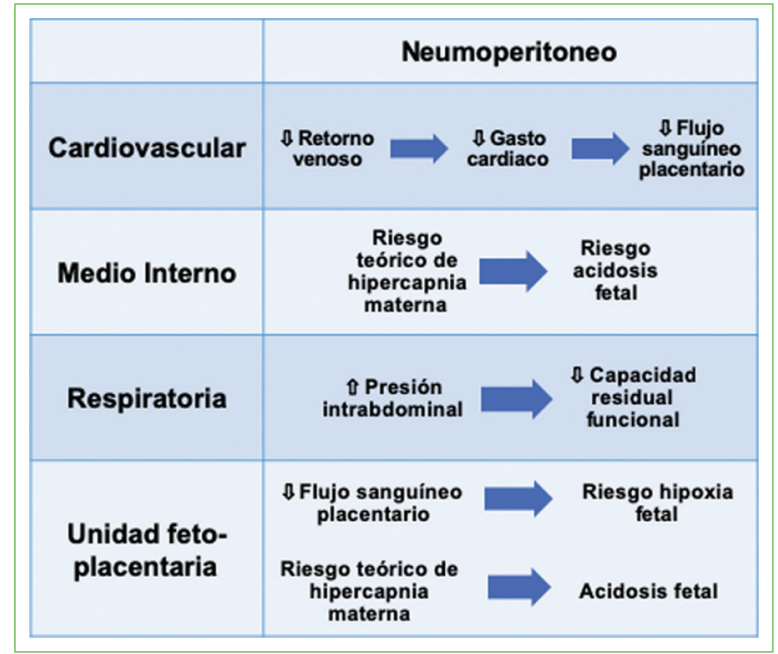

Figura 2. Efectos del neumoperitoneo en los diferentes sistemas ${ }^{26}$.

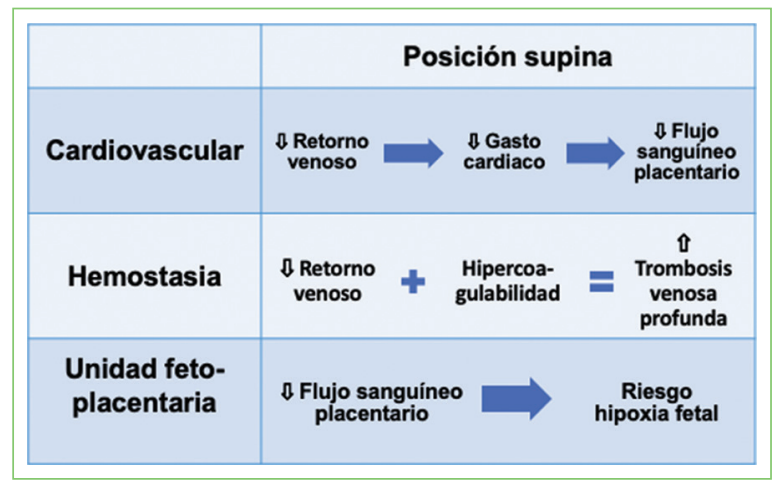

Figura 3. Efectos de la posición supina en los diferentes sistemas ${ }^{26}$.

- Mantener presiones intraabdominales $<12 \mathrm{mmHg}$ durante la cirugía (es posible aumentar hasta $18 \mathrm{mmHg}$ en el momento de la instalación de los trocares) ${ }^{36}$.

- En relación a la liberación de $\mathrm{CO}_{2}$ al utilizar la energía, este gas podría teóricamente alterar la oxigenación del feto, pero no está demostrado. De todas maneras, se recomienda evacuar el gas de forma eficiente mediante aspiración o liberación intermitente a través del trocar ${ }^{34}$.

- En embarazos del tercer trimestre, considerar el monitoreo fetal intraoperatorio, y si hay sospecha de sufrimiento fetal agudo, disminuir la presión intra-abdominal (PIA), realizar hiperventilación materna para $\mathrm{CO}_{2}$ espirado $32-34 \mathrm{mmHg}$, detener la manipulación de tejidos y considerar la necesidad de una cesárea de urgencia ${ }^{26,36}$.

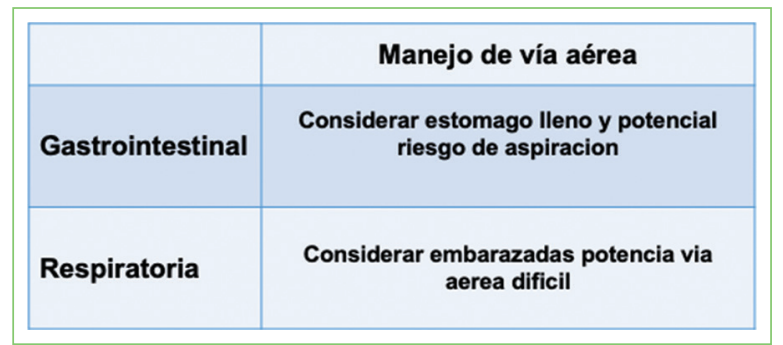

Figura 4. Consideraciones que tiene que tener en los diferentes sistemas el equipo de anestesia ${ }^{26}$.

\section{Posicionamiento de los trocares}

Dependerá de la edad gestacional y del tamaño del tumor anexial. En las figuras 5 a 7 se muestra cómo insertar los trocares según la edad gestacional:

- Embarazo menor de 12 semanas (Fig. 5):

- Paso 1: el acceso a la cavidad peritoneal, la insuflación y la introducción de la óptica se pueden realizar por el acceso umbilical $(1 \mathrm{~A}) \mathrm{o}$ el punto de Palmer (1B).

- Paso 2: la inserción de los tres trocares accesorios se realiza en las posiciones clásicas de la técnica laparoscópica, siendo dos en la fosa iliaca (derecha e izquierda) y uno en la mitad de la línea imaginaria entre la sínfisis púbica y el ombligo².

- Embarazo entre 12 y 20 semanas (Fig. 6):

- Paso 1: el acceso a la cavidad peritoneal, la insuflación y la introducción de la óptica se realizan por el punto de Palmer 1 .

- Paso 2: la inserción de los tres trocares accesorios se realiza uno a nivel umbilical y los otros dos en los flancos derecho e izquierdo en una línea imaginaria horizontal desde el ombligo².

- Embarazo mayor de 20 semanas (Fig. 7):

- Paso 1: el acceso a la cavidad peritoneal, la insuflación y la introducción de la óptica se realizan por el punto de Palmer'.

- Paso 2: la inserción de los tres trocares accesorios se realiza supraumbilical. La altura se ajusta según el tamaño uterino buscando mantener un margen de seguridad. Dos deben ir en los hipocondrios derecho e izquierdo ${ }^{2}$, y el tercero central en el epigastrio equidistante a los otros dos trocares accesorios.

Debemos fijarnos en el tamaño del tumor anexial. Si es menor de $10 \mathrm{~cm}$, se mantiene el posicionamiento según las semanas de embarazo, pero si es mayor podemos encontrar dos escenarios: que el tumor se 


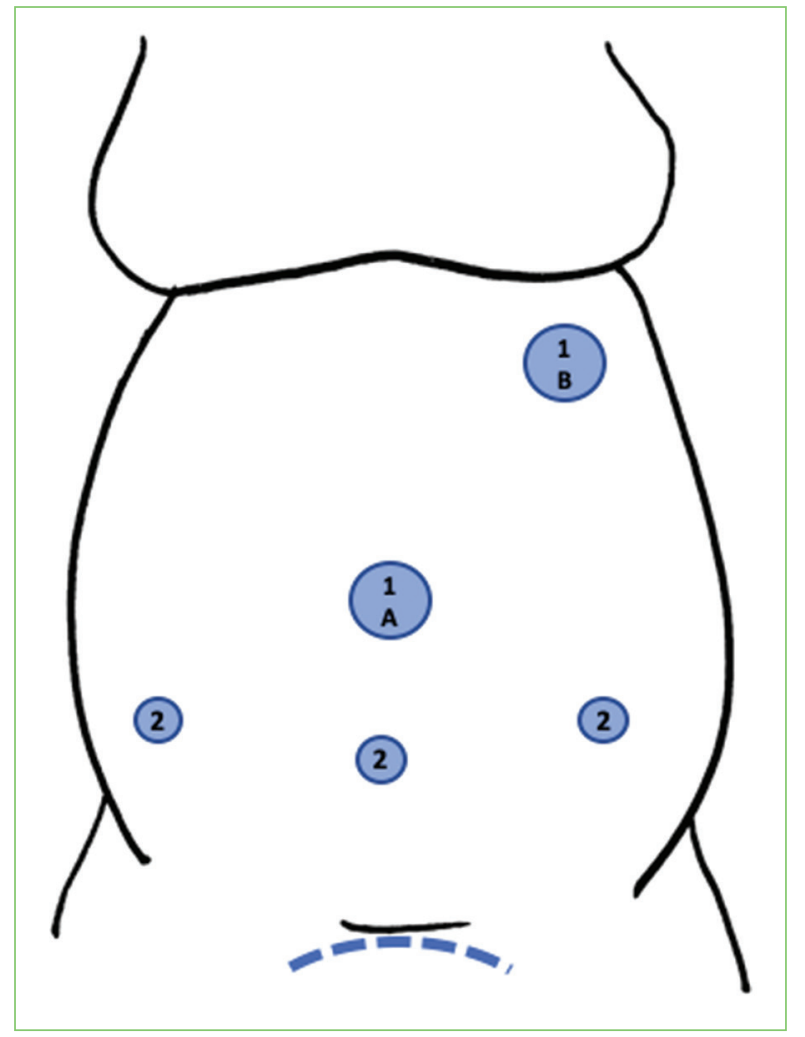

Figura 5. Paciente con embarazo menor a 12 semanas.

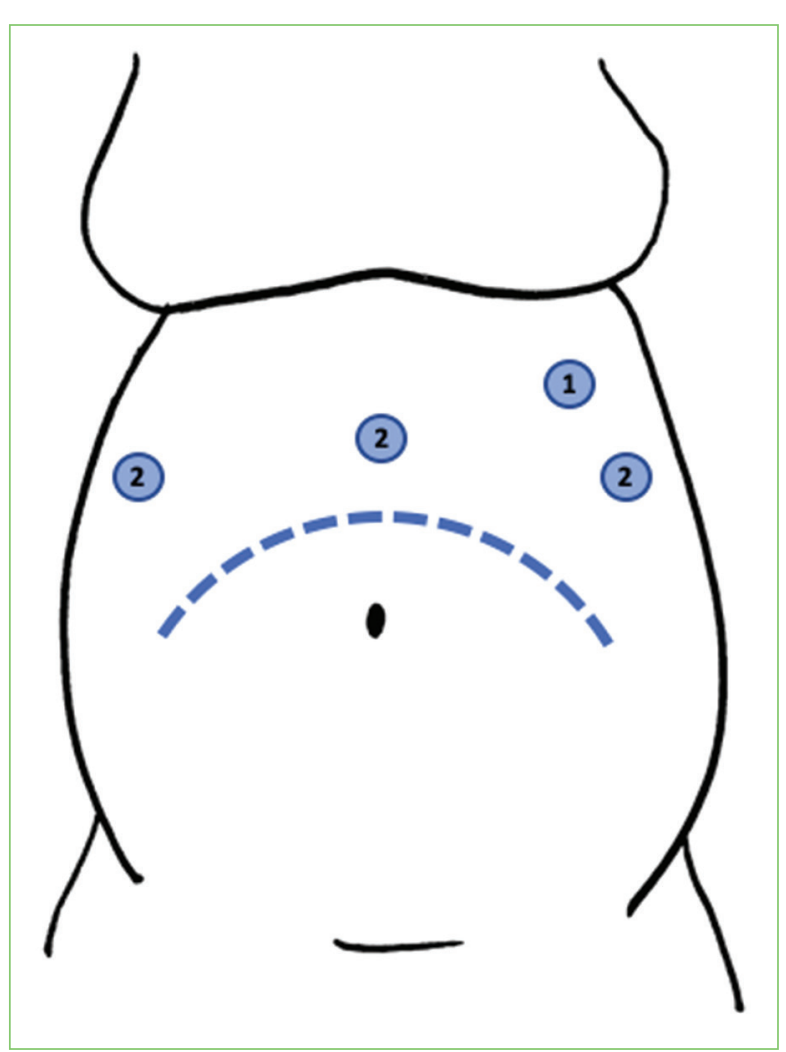

Figura 7. Paciente con embarazo mayor a 20 semanas.

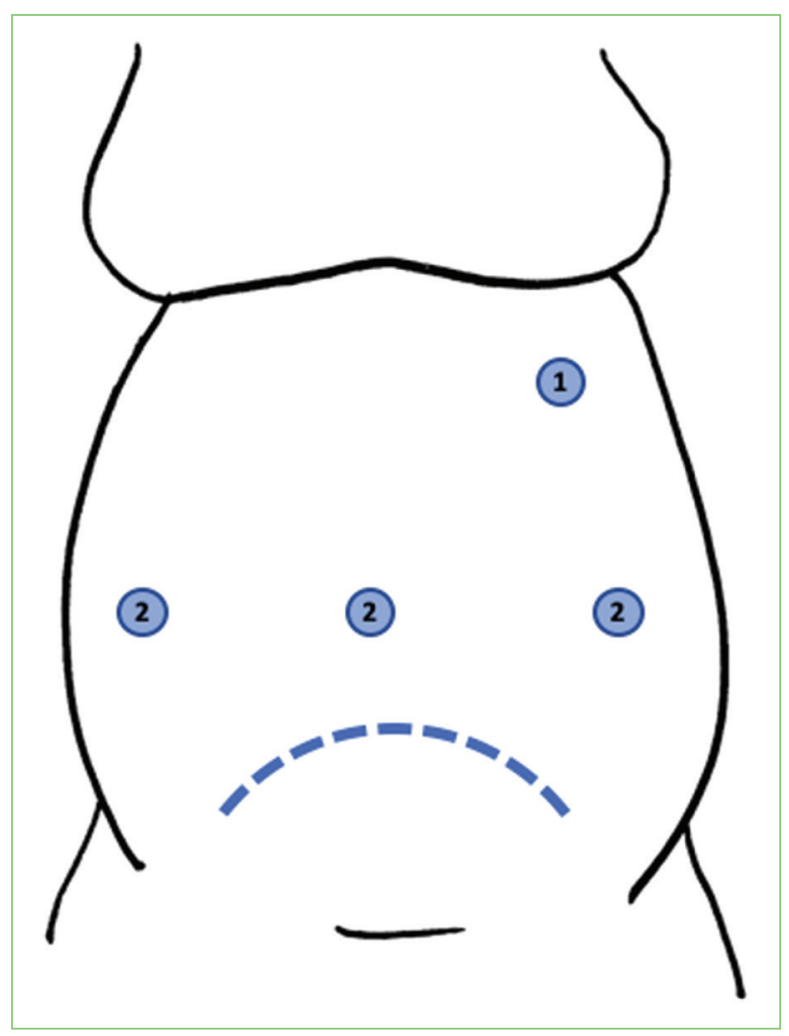

Figura 6. Paciente con embarazo entre 12 y 20 semanas. encuentre infraumbilical o supraumbilical, los cuales se exponen a continuación.

- Tumor mayor de $10 \mathrm{~cm}$ infraumbilical (Fig. 8) y supraumbilical (Fig. 9):

- Paso 1: el acceso a la cavidad peritoneal, la insuflación y la introducción de la óptica se realizan por el punto de Palmer1.

- Paso 2: la inserción de los tres trocares accesorios se realiza supraumbilical. La altura se ajusta según el tamaño del tumor anexial buscando mantener un margen de seguridad. Siempre uno debe ir al central y los otros dos se ajustan según el tamaño del tumor.

En la tabla 4 se indica la ubicación de los trocares según la edad gestacional y el tamaño del tumor anexial.

\section{Evidencias sobre el uso de medicamentos perioperatorios}

En relación al uso de progesterona posterior a una cirugía laparoscópica, en caso de realizar un abordaje previo al segundo trimestre, cuando es antes de las 8 semanas, se debe administrar progesterona, $200 \mu \mathrm{g}$ al día, hasta finalizar el primer trimestre. Entre las 8 y las 
Rev Chil Obstet Ginecol. 2021;86(6)

Tabla 4. Cómo abordar una lesión anexial según su tamaño y las semanas de gestación

\begin{tabular}{|c|c|c|c|}
\hline & Tumor anexial<10 cm & $\begin{array}{l}\text { Tumor anexial }>10 \mathrm{~cm} \\
\text { infraumbilical }\end{array}$ & $\begin{array}{l}\text { Tumor anexial }>10 \mathrm{~cm} \\
\text { supraumbilical }\end{array}$ \\
\hline Edad gestacional $>12$ semanas & $\begin{array}{l}\text { Según esquema de } \\
\text { embarazo }<12 \text { semanas }\end{array}$ & $\begin{array}{l}\text { Según esquema tumor } \\
\text { anexial }<10 \mathrm{~cm} \\
\text { supraumbilical }\end{array}$ & $\begin{array}{l}\text { Según esquema tumor } \\
\text { anexial }>10 \mathrm{~cm} \text { supraumbilical }\end{array}$ \\
\hline $\begin{array}{l}\text { Edad gestacional de } 12 \text { a } 20 \\
\text { semanas }\end{array}$ & $\begin{array}{l}\text { Según esquema de } \\
\text { embarazo de } 12 \text { a } 20 \\
\text { semanas }\end{array}$ & $\begin{array}{l}\text { Según esquema de embarazo } \\
\text { de } 12 \text { a } 20 \text { semanas o tumor } \\
\text { anexial }>10 \mathrm{~cm} \text { infraumbilical }\end{array}$ & $\begin{array}{l}\text { Según esquema de tumor } \\
\text { anexial }>10 \mathrm{~cm} \text { supraumbilical }\end{array}$ \\
\hline Edad gestacional $>20$ semanas & $\begin{array}{l}\text { Según esquema de } \\
\text { embarazo }>20 \text { semanas }\end{array}$ & $\begin{array}{l}\text { Según esquema de } \\
\text { embarazo }>20 \text { semanas }\end{array}$ & $\begin{array}{l}\text { Según esquema de embarazo }>20 \\
\text { semanas o tumor anexial }>10 \mathrm{~cm} \\
\text { supraumbilical (en base a cuál } \\
\text { alcanza un tamaño superior. }\end{array}$ \\
\hline
\end{tabular}

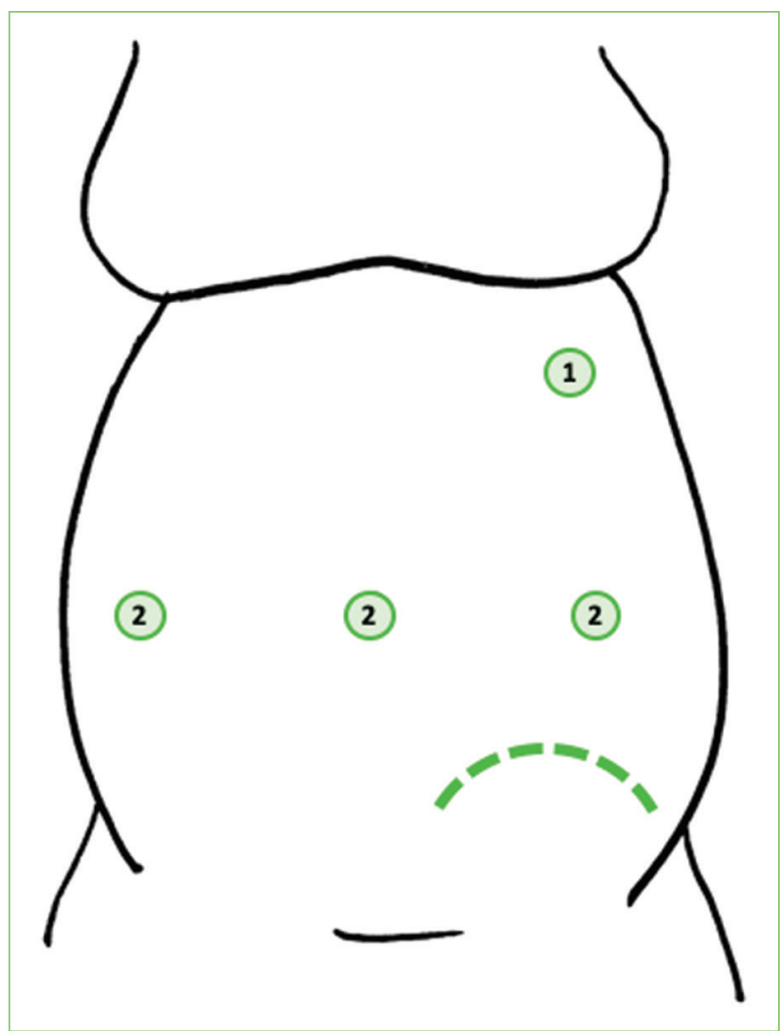

Figura 8. Tumor mayor a $10 \mathrm{~cm}$ infraumbilical.

10 semanas hay una transición de la producción de progesterona a la placenta, por lo que desde la semana 10 no hay suficiente evidencia para apoyar el uso de progesterona exógena ${ }^{37,38}$.

Si hablamos de la tocólisis perioperatoria, podría considerarse teóricamente su uso, pero la evidencia y el manejo actual en pacientes embarazadas que se realizarán una cirugía laparoscópica demuestran que no es necesario su uso sistemático, siendo la recomendación evaluar e individualizar la indicación caso a caso ${ }^{26}$.

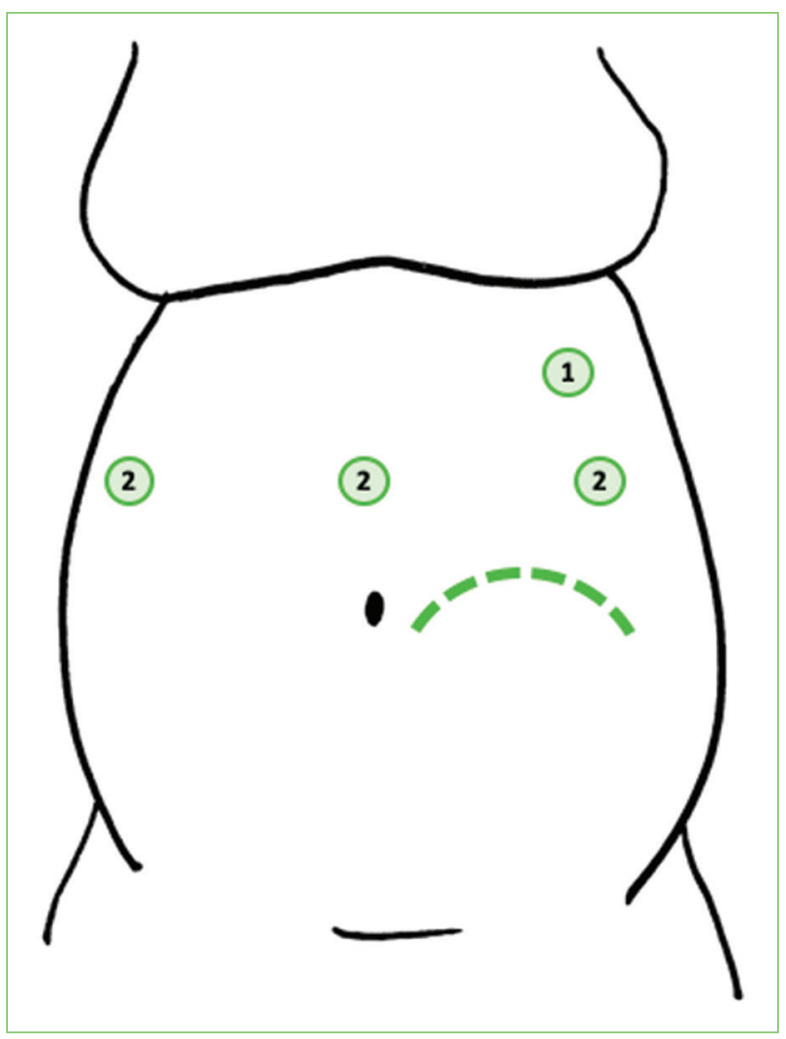

Figura 9. Tumor mayor a $10 \mathrm{~cm}$ supraumbilical.

\section{Complicaciones del procedimiento quirúrgico}

\section{Complicaciones tanto de la laparoscopia como la cirugía por laparotomía}

\section{INTRAOPERATORIAS}

Es el momento en que se presentan la mayor cantidad de complicaciones, sobre todo al ingresar a la cavidad peritoneal $^{26}$ : 
- Mayor riesgo de lesiones por el tamaño del útero grávido y la limitación de las maniobras instrumentales ${ }^{36}$.

- Mayor riesgo de hemorragia, debido al aumento de la vascularización uterina y anexial ${ }^{36}$.

- Mayor riesgo de perforación uterina ${ }^{36}$.

- Riesgo potencial de daño fetal ${ }^{36}$.

- Riesgo potencial de daño materno ${ }^{36}$.

\section{POSTOPERATORIAS}

- Mayor riesgo de aborto o de parto prematuro. La tasa de aborto es del $12-15 \%$ en el primer trimestre y del $5-6 \%$ en el segundo trimestre ${ }^{26}$. El riesgo de parto prematuro mayormente se da en el tercer trimestre, en un $15 \%{ }^{26}$.

- Mayor riesgo de enfermedades tromboembólicas por cambios fisiológicos en el embarazo: hipercoagulabilidad, lesión endotelial producto de la cirugía y la estasis venosa ${ }^{26}$.

- Riesgo de rotura prematura de membranas ${ }^{36}$.

- Riesgo de infecciones ${ }^{36}$.

- Riesgo de hernias ${ }^{36}$.

\section{Complicaciones asociadas únicamente a la laparoscopia}

- Riesgo de disminución del flujo útero-placentario asociado al aumento de la presión intraabdominal por el neumoperitoneo.

- Riesgo de dinámica uterina por el uso de electricidad y la manipulación del útero.

- Riesgo de complicaciones anestésicas por hipotensión asociada a los fármacos utilizados, así como también la hipercapnia que se puede producir y alteraciones del flujo útero-placentario asociado a acidosis fetal ${ }^{36}$.

\section{Conclusiones}

Los tumores anexiales son poco frecuentes en las embarazadas y en su mayoría suelen ser benignos. Es clave, tras realizar el diagnóstico, definir el riesgo de malignidad, el tipo de tumor y la presencia de síntomas, lo que llevará a tomar ciertas conductas. Es importante, como médicos, explicar las complicaciones y en conjunto evaluar el riesgo y el beneficio de realizar una intervención.

El manejo quirúrgico en caso de complicaciones puede realizarse por laparotomía o laparoscopia, siendo esta última una forma segura tanto para la madre como para el feto, que ha mostrado beneficios al compararse con la técnica abierta si es realizada por un equipo capacitado. Si se decide por una laparoscopia hay que tener consideraciones especiales en la técnica debido a los cambios fisiológicos de las embarazadas, la altura uterina y el tamaño de la lesión.

Cabe mencionar que, si bien existen estudios de laparoscopia en mujeres embarazadas, suelen ser en otras patologías, como colecistectomías o apendicectomías. Esta información se podría extrapolar a las lesiones anexiales, pero faltan estudios.

\section{Financiamiento}

No se obtuvo financiamiento para este estudio.

\section{Conflicto de intereses}

No se presentan conflictos de intereses.

\section{Responsabilidades éticas}

Protección de personas y animales. Los autores declaran que para esta investigación no se han realizado experimentos en seres humanos ni en animales.

Confidencialidad de los datos. Los autores declaran que han seguido los protocolos de su centro de trabajo sobre la publicación de datos de pacientes.

Derecho a la privacidad y consentimiento informado. Los autores han obtenido el consentimiento informado de los pacientes y/o sujetos referidos en el artículo. Este documento obra en poder del autor de correspondencia.

\section{Bibliografía}

1. Hoover K, Jenkins TR. Evaluation and management of adnexal mass in pregnancy. Am J Obstet Gynecol. 2011;205:97-102.

2. Glanc $P$, Salem S, Farine D. Adnexal masses in the pregnant patient: a diagnostic and management challenge. Ultrasound Q. 2008;24:225-40.

3. Ye P, Zhao N, Shu J, Shen H, Wang Y, Chen L, et al. Laparoscopy versus open surgery for adnexal masses in pregnancy: a meta-analytic review. Arch Gynecol Obstet. 2019;299:625-34.

4. Yuen PM, Ng PS, Leung PL, Rogers MS. Outcome in laparoscopic management of persistent adnexal mass during the second trimester of pregnancy. Surg Endosc. 2004;18:1354-7.

5. Mathevet P, Nessah K, Dargent D, Mellier G. Laparoscopic management of adnexal masses in pregnancy: a case series. Eur J Obstet Gynecol Reprod Biol. 2003:108:217-22.

6. Biggs WS, Marks ST. Diagnosis and management of adnexal masses Am Fam Physician. 2016;93:676-81.

7. Woo-Chul K, Yong-Soon K. Laparoendoscopic single-site surgery for exteriorization and cystectomy of an ovarian tumor during pregnancy. J Minim Invasive Gynecol. 2010;17:386-9.

8. Webb KE, Sakhel K, Chauhan SP, Abuhamad AZ. Adnexal mass during pregnancy: a review. Am J Perinatol. 2015;32:1010-6.

9. Schmeler KM, Mayo-Smith WW, Peipert JF, Weitzen S, Manuel MD, Gordinier ME. Adnexal masses in pregnancy: surgery compared with observation. Obstet Gynecol. 2005;105:1098-103. 
10. Glanc $P$, Salem $S$, Farine D. Adnexal masses in the pregnant patient: a diagnostic and management challenge. Ultrasound Q. 2008;24:225-40.

11. Hakoun AM, Abou-Al-Shaar I, Zaza KJ, Abou-Al-Shaar H, A Salloum MN Adnexal masses in pregnancy: an updated review. Avicenna J Med. 2017;7:153-7.

12. Aggarwal $P$, Kehoe $S$. Ovarian tumours in pregnancy: a literature review. Eur J Obstet Gynecol Reprod Biol. 2011;155:119-24.

13. Goh W, Bohrer J, Zalud I. Management of the adnexal mass in pregnancy. Curr Opin Obstet Gynecol. 2014;26:49-53.

14. Grimm D, Woelber L, Trillsch F, Keller-v Amsberg G, Mahner S. Clinical management of epithelial ovarian cancer during pregnancy. Eur J Cancer. 2014;50:963-71.

15. Moore RD, Smith WG. Laparoscopic management of adnexal masses in pregnant women. J Reprod Med. 1999;44:97-100.

16. Leiserowitz GS, Xing G, Cress R, Brahmbhatt B, Dalrymple JL, Smith LH Adnexal masses in pregnancy: how often are they malignant? Gynecol Oncol. 2006;101:315-21.

17. Cooper L, Patounakis G, Rosenblum N. Vincenzo Berghella. Capítulo 32 The adnexal mass, 343. Libro: Obstetric Evidence Based Guidelines, Third Edition, Journal of Maternal Fetal \& Neonatal Medicine, Philadelphia, USA. Editorial: CRC Press, 2017.

18. Nunes N, Ambler G, Foo X, Naftalin J, Widschwendter M, Jurkovic D. Use of IOTA simple rules for diagnosis of ovarian cancer: meta-analysis. Ultrasound Obstet Gynecol. 2014;44:503-14.

19. De Haan J, Verheecke M, Amant F. Management of ovarian cysts and cancer in pregnancy. Facts Views Vis Obgyn. 2015;7:25-31.

20. Gupta D, Lis CG. Role of CA125 in predicting ovarian cancer survival - a review of the epidemiological literature. J Ovarian Res. 2009:9:13.

21. Ortega X, Silva C. Radiología diagnóstica en la embarazada: consideraciones sobre exposición a la radiación y riesgo del feto. Clínica Las Condes. Departamento de diagnóstico por imágenes. Rev Med Clin Las Condes. 2008:19:298-303.

22. Kotecha HM, Mclntosh LJ, Lo HS, Chen BY, Dupuis CS. What to expect when they are expecting: magnetic resonance imaging of the acute abdomen and pelvis in pregnancy. Curr Probl Diagn Radiol. 2017:46:423-31.

23. Yacobozzi M, Nguyen D, Rakita D. Adnexal masses in pregnancy. Semin Ultrasound CT MR. 2012;33:55-64.

24. Niloff JM, Knapp RC, Schaetzi E, Reynolds C, Bast RC Jr. CA125 antigen levels in obstetric and gynecology patients. Obstet Gynecol. $1984 ; 64: 703-7$
25. Yen CF, Lin SL, Murk W, Wang CJ, Lee CL, Soong YK, et al. Risk analysis of torsion and malignancy for adnexal masses during pregnancy. Fertil Steril. 2009;91:1895-902.

26. Larraín D, Durruty G, Pomés C, Cuello M. Consideraciones para el uso de la laparoscopia durante el embarazo. Rev Chil Obstet Ginecol. 2007;72:247-57

27. Ávila M, Zavala C, Coronel F. Colecistectomía laparoscópica en pacientes embarazadas: experiencia del Hospital General de México 20082012. Ginecol Obstet Mex. 2014;82:509-17.

28. Hernández A, Aguirre X, Pedraza L. Colecistectomía laparoscópica en el embarazo. Experiencia de cinco años en el Hospital Español de México y revisión de la bibliografía. Ginecol Obstet Mex. 2011;79:200-5.

29. John P, Curtin MD. Management of the adnexal mass. Gynecol Oncol. 1994:55:42-6

30. Tratamiento laparoscópico de las masas anexiales. Progresos de Obstetricia y Ginecología, 49(7), 410-414. Protocolo n. 07 endoscopio ginecología.

31. Oelsner G, Shashar D. Adnexal torsion. Clin Obstet Gynecol. 2006:49:459-63.

32. Zamboni M, Palominos G, Núñez F, Durruty G, Mayerson D, Barrena N, et al. Manejo conservador de la torsión anexial: ¿una alternativa o una obligación frente a un posible error de apreciación por parte del cirujano? Rev Chil Obstet Ginecol. 2011;76:248-56.

33. Steinbrook RA, Bhavani-Shankar K. Hemodynamics during laparoscopic surgery in pregnancy. Anesth Analg. 2001;93:1570-1.

34. Stepp K, Falcone T. Laparoscopy in the second trimester of pregnancy. Obstet Gynecol Clin North Am. 2004;31:485-96.

35. Geerts WH, Pineo GF, Heit JA, Bergqvist D, Lassen MR, Colwell CW, et al. Prevention of venous thromboembolism: the Seventh ACCP Conference on Antithrombotic and Thrombolytic Therapy. Chest. 2004;126(3 Suppl):338-400.

36. Ball E, Waters N, Cooper N, Talati C, Mallick R, Rabas S, et al. Evidence-based guideline on laparoscopy in pregnancy: commissioned by the British Society for Gynaecological Endoscopy (BSGE) endorsed by the Royal College of Obstetricians \& Gynaecologists (RCOG). Facts Views Vis Obgyn. 2019:11:5-25.

37. Csapo Al, Pulkkinen MO, Ruttner B, Sauvage JP, Wiest WG. The significance of the human corpus luteum in pregnancy maintenance. I. Preliminary studies. Am J Obstet Gynecol. 1972;112:1061-7.

38. Csapo Al, Pulkkinen MO, Wiest WG. Effects of luteectomy and progesterone replacement therapy in early pregnant patients. Am J Obstet Gynecol. 1973;115:759-65 